\title{
Algorithmic Approach to Star Partition of the Graph
}

\author{
Ishwar Baidari \\ Asst. Professor \\ Dept of Computer Science \\ Karnatak University,Dharwad
}

\author{
H B Walikar, PhD. \\ Professor \\ Dept of Computer Science \\ Karnatak University,Dharwad
}

\author{
Shridevi Shinde \\ Research Scholar \\ Dept of Computer Science \\ Karnatak University,Dharwad
}

\begin{abstract}
The purpose of this paper is to design an algorithm for star partitions of the graph. We shall now bring out a useful connection between the domination number of a graph and what we shall choose to call the 'star partition number' of the graph which is an invariant of the graph defined by a certain type of partition of its vertex set. We consider finite undirected graphs without loops or multiple edges
\end{abstract}

\section{Keywords}

star partition, domination number, multiple edges,

\section{INTRODUCTION}

Let $G$ be a graph and $P=\left\{v_{1}, v_{2}, \ldots ., v_{t}\right\}$ be a partition of $V(G)$ - we then say that $P$ is a partition of $G$. Denoted by $\pi(G)$ the set of all partitions of $G$. A star partition of $G$ is a partition $P=\left\{v_{1}, v_{2}, \ldots . ., v_{t}\right\}$ of $\mathrm{G}$ such that $v_{i}$ is a star for each $i \in\left\{1,2, \ldots, v_{t}\right\}$ of graph $\mathrm{G}$ has a star partition, viz $P_{o}=\{\{u\} / u \in V(G)\}$, the trivial star partition of $G[1,3,6$, $7,8,9,13,15]$. Thus, the set $\pi^{*}(G)$ of all star partition of $G$ is nonvoid. The number $\gamma^{*}(G)=\min _{P \in \pi^{*} P(G)}|P|$ is called the star partition number of $G$. The members of the set $\pi^{*}(G)=$ $\left\{P \in \pi^{*}(G) /|P|=\gamma^{*}(G)\right\}$ are called the minimum star partition of $G$. H. B. Walikar [1] has proved following preposition and theorems.

\section{PROPOSITION}

For any graph $\boldsymbol{G}, \boldsymbol{\gamma}(\boldsymbol{G}) \leq \boldsymbol{\gamma}^{*}(\boldsymbol{G})$.

There are graphs $G$ for which $\gamma(G)=\gamma *(G)$ and in the following proposition we shall show that trees fall under this category.

\section{PROPOSITION}

\section{For any tree $\mathrm{T}, \gamma(T)=\gamma^{*}(T)$.}

3.1 Remark: The star partition constructed in the proof of the above proposition is not unique even for a given minimum dominating set containing all the supports in $\boldsymbol{T}$. We are now ready to give a characterization of tree having a unique minimum dominating set.

Henceforth, given $P=\left\{v_{1}, v_{2}, \ldots . ., v_{t}\right\} \in \pi^{*}(G)$ we let $v_{i}$ denote the centre of the $\operatorname{star}\left\langle v_{i}\right\rangle, i \in\{1,2, \ldots \ldots, t\} ; c=$ $\left\{v_{1}, v_{2}, \ldots ., v_{t}\right\}$ and let $c_{j}=c-\left\{v_{j}\right\}$ for any $j \in$ $\{1,2, \ldots \ldots, t\}$.

\subsection{Theorem 1}

A tree $\boldsymbol{T}$ has a unique minimum dominating set if and only if for every $\boldsymbol{P}=\left\{\boldsymbol{v}_{\mathbf{1}}, \boldsymbol{v}_{2}, \ldots, \boldsymbol{v}_{\boldsymbol{t}}\right\} \in \boldsymbol{\pi}^{*}(\boldsymbol{G})$, and for every $i \in\{1,2, \ldots \ldots, t\}, u \in N\left(v_{i}\right) \cap v_{i}$ implies that there exists $w \in\left(N\left(v_{i}\right)-N(u) \cap v_{i}\right.$ with $N(w) \cap c_{i}=\emptyset$.

\subsection{COROLLARY}

The path $P_{n}$ of length $\mathrm{n}$ has unique minimum dominating set if, and only if $n \equiv 2(\bmod 3)$. Notice from the proof of theorem 1 that it does not make use of any property of a tree $T$, other than that of $\gamma^{*}(T)=\gamma(T)$. Thus, the following extension of theorem 1 also holds, where $v_{i}, c$ and $c_{i}$ have the same meaning as above.

\subsection{Theorem 2}

Let $G$ be any graph such that $\gamma^{*}(T)=\gamma(T)$. Then $G$ has a unique minimum dominating set if, and only if for every $P=\left\{v_{1}, v_{2}, \ldots ., v_{t}\right\} \in \pi^{*}(G)$ and $\quad$ for $\quad$ every $i \in$ $\{1,2, \ldots, t\}, u \in N\left(v_{i}\right) \cap v_{i}$ implies that there exists $w \in\left(N\left(v_{i}\right)-N(u) \cap v_{i}\right.$ with $N(w) \cap c_{i}=\emptyset$.

We already introduced the star partition number $\gamma^{*}(G)$ of a graph $G$ - as the minimum order of the partition of the vertex set of $G$ into subsets, each of which induces a star in $G$.Here, we consider the detail study of the star partition number and extend the result $\gamma^{*}(T)=\gamma(T)$ for a triangle - free graph.

For some standard graphs, the star - partition number can be easily found are given as follows:

$$
\begin{aligned}
& \text { 1) } \gamma^{*}\left(K_{p}\right)=\left\{\frac{p}{2}\right\} \\
& \text { 2) } \gamma^{*}\left(K_{m, n}\right)=\left\{\begin{array}{rr}
1 & \text { if either } m \text { or } n=1 \\
2 & \text { if } m, n \geq 2
\end{array}\right. \\
& \text { 3) } \gamma^{*}\left(C_{p}\right)=\left\{\begin{array}{lr}
2 \text { if } & p=3 \\
\left\{\frac{p}{3}\right\} & \text { if } p \geq 4
\end{array}\right. \\
& \text { 4) } \gamma^{*}\left(P_{k}\right)=\left\{\frac{k+1}{3}\right\}
\end{aligned}
$$

Where $\{X\}$ denote the smallest the integer not smaller than the real number $x$.

We know that $\gamma^{*}(T)=\gamma(T)$ for any tree $T$. It has its own importance in characterizing the trees having unique minimum dominating sets. Moreover, the minimum star partition (whose order equals to the domination number). The following theorem extends this result to the graphs having no triangles) i.e. a cycle of length three.)

\subsection{Theorem 3}

For any triangle - free graph $G, \gamma^{*}(G)=\gamma(G)$

\subsection{Corollary}

For any tree $T, \gamma^{*}(T)=\gamma(T)$

For a graph $G$ we denote by $G^{+}$the graph obtained from $G$ by the adjunction of a new adjacencies $V^{\prime}$ for every vertex $V$ in $G$ and making new adjacencies $V V^{\prime}$ for every vertex $V$ in $G$.

\subsection{Proposition}

Every graph $H$ can be imbedded as an induced sub graph of a graph $G$ for which $\gamma^{*}(G)=\gamma(G)$. 


\subsection{Corollary}

There is no finite family of forbidden graphs by which the class $G=\left\{H / \gamma^{*}(H)=\gamma(H)\right\}$ is characterized.

This Corollary shows that the results of the sort of Theorem 3 are infinite in number.

The following result gives the bounds on the size of a graph $G$ of order $p$ with given star partition number. It is well known that "the minimum number of edges in a graph $G$ of order $p$, having $k$ components, has size $p-k$ if, and only if each component of $g$ is a tree".

Let $p=\left\{v_{1}, v_{2}, \ldots \ldots, v_{k}\right\} \in \pi^{*}(G)$, $\operatorname{Then} \gamma^{*}(G)=$

$|P|$ and let $\left|v_{i}\right|=p_{i}$, for each $i \in\{1,2, \ldots, k\}$.

\subsection{Proposition}

Let $G$ be a $(p, q)$-graph with $\gamma^{*}(G)=k$. Then

$$
p-k \leq q \leq \frac{1}{2}\left(p^{2}-\sum_{i=1}^{k} p_{i}^{2}\right)+p-k
$$

Furthermore, the lower bound in (1) attains if, and only if $G=\bigcup_{i=1}^{k} K_{1}, p_{i}-1$ and the upper bound in (1) attains if, and only if $G=\sum_{i=1}^{k} K_{1}, p_{i}-1$, where pi are as defined above and ' $\sum$ ' denotes the recursive graph 'join'.

\subsection{Proposition}

Let $p, q$ and $k$ be any three positive integers and $p=$ $\sum_{i=1}^{k}(p i)$ where $p_{i} \geq 0$. For each $i \in\left\{v_{1}, v_{2}, \ldots, k\right\}$, then for every integer $q$ with $p-k \leq q \leq \frac{1}{2}\left(p^{2}-\sum_{i=1}^{k} p_{i}^{2}\right)+p-k$ there exists a graph $G$ with $p$ vertices and $q$ edges satisfying $\gamma^{*}(G)=k$.

The following results deal with the Cartesian product of two graphs and star partition number. Let $G_{1}$ and $G_{2}$ be two graph, the Cartesian product $G_{1} \times G_{2}$ is the graph whose vertex set $V\left(G_{1} \times G_{2}\right)=V\left(G_{1}\right) \times\left(G_{2}\right)$ and the edge set $E(G 1 \times$ $G 2)=\left\{\left\{\left(a_{i}, b_{i}\right),\left(a_{j}, b_{j}\right)\right\} /\right.$ either $a_{i}=$ $a_{j}$ and $b_{i}$ is adjacent to $b_{j}$ or $a_{i}$ is adjacent to $a_{j}$ and $b_{i}=$ $\left.b_{j}\right\}$, Where $V\left(G_{1}\right)=\left\{a_{1}, a_{2}, \ldots . a_{p_{1}}\right)$ and $v\left(G_{2}\right)=$ $\left\{b_{1}, b_{2}, \ldots \ldots b_{p_{2}}\right\}$.

\subsection{Proposition}

Let $G_{1}$ and $G_{2}$ be any two graphs of order $P_{1}$ and $P_{2}$ respectively. Then $\gamma^{*}\left(G_{1} \times G_{2}\right) \leq \min \left\{p_{1} \gamma^{*}\left(G_{2}\right) \cdot P_{2} \gamma^{*}\left(G_{1}\right)\right\}$

\subsection{Algorithm: Star Partition $[G]$}

1. For each vertex $u \in V[G]$.

2. If $N(u)$ is independent set

3. Then do $v=v-N[u]$

4. Else choose an independent subsets $S$ of $N(u)$

5. And set $V=V-S \cup\{u\}$

6. Repeat 1 until $V=\emptyset$.

The procedure star partition works as follows: Line 1 consider each vertex in the graph. Line 2 checks the open neighborhood of $N(u)$ is independent set or not. Line 3 if the independent set condition is satisfied then it subtract closed neighborhood set $N[u]$ from vertex set $V . V-\boldsymbol{S} \cup\{u\}$. In line 4 if the independent set is not satisfied it choose subset $S$ of $N(u)$ and set $V=V-S \cup\{u\}$. This process will repeat until $V=\varnothing$.
Example: Consider the following graph for star partition

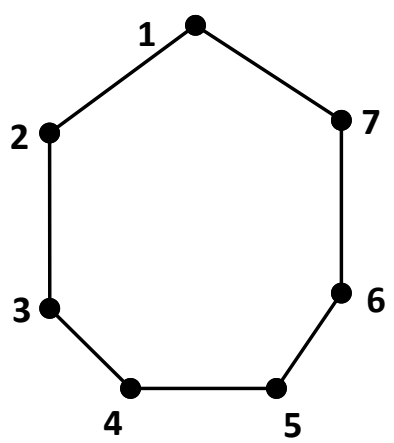

$V=\{1,2,3,4,5,6,7\}$

$$
\begin{aligned}
\text { Step } 1 \text { Let } u & =1, N(1)=\{2,7\} \\
V & =V-N[1] \\
V & =\{1,2,3,4,5,6,7\}-\{1,2,7\} \\
V & =\{3,4,5,6\}
\end{aligned}
$$

Step 2 Next vertex $u=3, N(3)=\{\{2,4\}-N(1)\}$

$$
\begin{aligned}
& =\{4\} \\
V & =\{3,4,5,6\}-\{3,4\} \\
V & =\{5,6\}
\end{aligned}
$$

$$
\begin{aligned}
& \text { Step } 3 u=5, N(5)=\{4,6\}-N(3) \\
& =\{6\} \\
& V=\{5,6\}-\{5,6\}=\emptyset
\end{aligned}
$$

Step $4 V=\varnothing$

Then the star partition of the graph is as follows

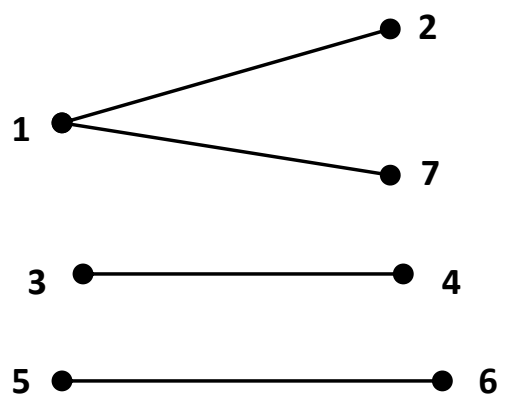

The star partition set is $\{\{1,2,7\},\{3,4\},\{5,6\}\}$

\section{Conclusion}

Here, we raise the problem of determining the structure of graphs $G$ having one and only one minimum dominating set $D$, and determine the structure of such trees in terms of certain partitions, called star partition of $G$ of the vertex set of $G$. We developed the algorithm to handle the star partition of $G$ in linear time.

\section{References}

[1] H B Walikar, "Some Topics in Graph Theory (Contribution to the Theory of Domination in Graphs and its Applications", 1980.

[2] E Sampathkumar and H B Walikar "The Connected Domination Number of Graph", Jl.Maths.Phy.Sci.13(6):1979,607-613. 
[3] C Berge, The Theory of Graph and its Applications. Metuen, London, 1962.

[4] E. J Cockayne, and S.T.Hedetniemi, Towards a theory of domination in graphs. Networks, 7 (1977), 247- 261.

[5]. Teresa W.Haynes, Stephen T. Hedetniemi and Peter J. Slater "Fundamentals of Domination in Graphs". Pure and Applied Mathematics. Marcel and Dekker 1998.

[6]. Douglas B. West "Introduction to Graph Theory" PHI, $2^{\text {nd }}$ edition 2001.

[7] Thomas H. Cormen, Charles E. Leiserson and Ronald L. Rivest "Introduction to Algorithms", PHI, Fourth Printing 2001.

[8]. Gary Chartrand and Ortrud. R Oellermann "Applied and Algorithmic Graph Theory", McGraw-Hill, International edition 1993.

[9] J A Bondy and U S R Murthy "Graph Theory", Springer2008.

[10] D E Knuth "Fundamental Algorithm Volume 1"Addision Wesely Publishing Company,Second printing 1969.
[11] E. J Cockayne, S E Goodman, and S.T.Hedetniemi, "A Linear Algorithm for the Domination Number of Tree", Inform.Process.Lett.,4:41-44,1975.

[12] S L Mitchell, E.J.Cockayne, And S T Hedetniemi. "Linear Algorithms on Recursive Representations of trees", J.Comput.System Sci.,18(1):76-85,1979.

[13] F Harary, Graph Theory, Addison - Wesley, Reading, Mass, 1969.

[14] F. Harary, R.W. Robinson and N.C.Wormald, Isomorphic factorization - I: Complete Graphs. Trans. Amer. Math. Soc., 242, 1978, 243-260.

[15] O.Ore, Theory of Graphs, Amer. Math. Soc. Colloq. Pub., Providence, RI 38,1962.

[16].Cockayne, E.J.and S.T.Hedetniemi, A linear algorithm for the maximum weight of an independent set in a tree.In Proc.Seventh S.E Conf. on Combinatorics, Graph. Theory and Computing, pages217-228.Utilities Math., Winnipeg, 1976.

[17].Cockayne, E.J.and S.T.Hedetniemi, Towards a Theory Domination in graphs. Networks, 1977

[18] E. J Cockayne, .and S.T.Hedetniemi, Towards a theory of domination in graphs. 